\title{
Onset of Entanglement and Noise Cross-Correlations in Two-Qubit System Interacting with Common Bosonic Bath
}

\author{
Vladimir Privman, Dmitry Solenov, and Denis Tolkunov \\ Department of Physics, Clarkson University, Potsdam, New York 13699, USA \\ E-mails: Privman@clarkson.edu, Solenov@clarkson.edu, and Tolkunov@clarkson.edu
}

\begin{abstract}
We summarize our recent results [1,2] for the induced exchange interaction due to thermal bosonic environment (bath) which also generates quantum noise. Our focus here is on the onset of the interaction. We demonstrate that the induced interaction can be used to manipulate and create entanglement over time scales sufficiently large for controlling the two-qubit system for quantum computing applications, though ultimately the noise effects will dominate.
\end{abstract}

\section{Introduction}

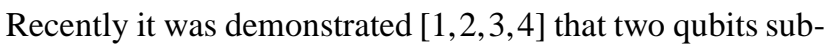
ject to common thermal bosonic environment (bath) can develop considerable entanglement. A similar result has also been obtained for qubits interacting via fermionic environment [5]. Here we review our results on the derivation of the induced exchange interaction and quantum noise in a unified formulation [1] 2], focusing the presentation on the onset and development of the cross-qubit correlations due to the bath.

We consider a $1 \mathrm{D}$ channel model for the bath, motivated by recent experiments [6], and allow bosons (e.g. phonons, photons) to propagate along a single direction with wave vector $k$ and dispersion $\omega_{k}=c_{s} k$. More general results are available in [2]. Two qubits immersed in this environment are separated by distance $r_{2}-r_{1}=|\mathbf{d}|$ such that the interaction due to the wave function overlap is negligible. The qubits' interaction with the bosonic bath is introduced [1 7] as

$$
H_{S B}=\sum_{j=1,2} \sigma_{x}^{j} X_{m}^{j}
$$

where $\sigma_{x}^{j}$ is the standard Pauli matrix of qubit $j=1$ and 2, and

$$
X_{m}^{j}=\sum_{k} g_{k}^{m}\left(a_{k} e^{i k r_{j}}+a_{k}^{\dagger} e^{-i k r_{j}}\right) .
$$

The total Hamiltonian is $H=H_{S}+H_{B}+H_{S B}$, where $H_{B}=\sum_{k} \omega_{k} a_{k}^{\dagger} a_{k}, H_{S}$ represents the Hamiltonian of the qubit system, and we set $\hbar=1$. The reduced density matrix that describes the dynamics of the qubit system is, then, given as the trace of the total density matrix over the bath modes,

$$
\rho_{S}(t)=\operatorname{Tr}_{B}\left(e^{-i H t} \rho_{S}(0) \rho_{B} e^{i H t}\right),
$$

where the initial density matrix is assumed factorized and consists of the system and bath parts. The latter is $\rho_{B}=$ $e^{-H_{B} / k T} / \operatorname{Tr}_{B}\left(e^{-H_{B} / k T}\right)$. For large times, a more realistic model of the environment assumes rethermalization, and Markovian schemes are appropriate for the description of the dynamics [2]. However, for short times the present formulation is adequate and provides a useful solvable model for the case of otherwise gapless qubits, $H_{S}=0$, which we consider from now on.

\section{Exact solution to the reduced density matrix}

With the assumptions outlined above, we utilized bosonic operator techniques [1] to derive an exact expression

$$
\rho_{S}(t)=\sum_{\lambda, \lambda^{\prime}} P_{\lambda} \rho_{S}(0) P_{\lambda^{\prime}} e^{\mathfrak{L}_{\lambda \lambda^{\prime}}(t)}
$$

Here the projection operator is defined as $P_{\lambda}=$ $\left|\lambda_{1} \lambda_{2}\right\rangle\left\langle\lambda_{1} \lambda_{2}\right|$, with $\left|\lambda_{j}\right\rangle$ the eigenvectors of $\sigma_{x}^{j}$. The real part of the exponent in (4) leads to decay of off-diagonal density-matrix elements resulting in decoherence,

$$
\begin{aligned}
\operatorname{Re} \mathfrak{L}_{\lambda \lambda^{\prime}}(t) & =-\sum_{k} G_{k}(t, T)\left[\left(\lambda_{1}^{\prime}-\lambda_{1}\right)^{2}+\left(\lambda_{2}^{\prime}-\lambda_{2}\right)^{2}\right. \\
& \left.+2 \cos \left(\frac{\omega_{k}|\mathbf{d}|}{c_{s}}\right)\left(\lambda_{1}^{\prime}-\lambda_{1}\right)\left(\lambda_{2}^{\prime}-\lambda_{2}\right)\right]
\end{aligned}
$$

The imaginary part, yielding the induced interaction, is

$$
\operatorname{Im} \mathfrak{L}_{\lambda \lambda^{\prime}}(t)=\sum_{k} C_{k}(t) \cos \left(\frac{\omega_{k}|\mathbf{d}|}{c_{s}}\right)\left(\lambda_{1} \lambda_{2}-\lambda_{1}^{\prime} \lambda_{2}^{\prime}\right) .
$$

We defined the standard "spectral" functions [7, 8]

$$
G_{k}(t, T)=2 \frac{\left|g_{k}\right|^{2}}{\omega_{k}^{2}} \sin ^{2}\left(\frac{\omega_{k} t}{2}\right) \operatorname{coth}\left(\frac{\omega_{k}}{2 k_{B} T}\right),
$$




$$
C_{k}(t)=2 \frac{\left|g_{k}\right|^{2}}{\omega_{k}^{2}}\left(\omega_{k} t-\sin \omega_{k} t\right) .
$$

To evaluate (5) and (6), we consider the model in which the density of modes together with the coupling constants are approximated by the power-law function of the frequency with superimposed exponential cutoff [7], i.e.,

$$
\sum_{k}\left|g_{k}\right|^{2} \rightarrow \alpha_{n} \int_{0}^{\infty} d \omega \omega^{n} \exp \left(-\omega / \omega_{c}\right)
$$

For $n=1$ this corresponds to the well known Ohmic model [7].

\section{Induced cross-qubit interaction and noise}

One can show that if the real part of $\mathfrak{L}_{\lambda \lambda^{\prime}}(t)$ were absent, the exponential involving the imaginary part would yield coherent dynamics with the unitary evolution operator $\exp \left[-i\left(H_{\text {int }}+F(t)\right) t\right]$. The constant Hamiltonian $H_{\text {int }}$ represents the induced interaction,

$H_{\mathrm{int}}=-\frac{2 \alpha_{n} \Gamma(n) c_{s}^{n} \omega_{c}^{n}}{\left(c_{s}^{2}+\omega_{c}^{2}|\mathbf{d}|^{2}\right)^{n / 2}} \cos \left[n \arctan \left(\frac{\omega_{c}|\mathbf{d}|}{c_{s}}\right)\right] \sigma_{x}^{1} \sigma_{x}^{2}$.

The time dependent term is given by

$$
F(t)=2 \sigma_{x}^{1} \sigma_{x}^{2} \alpha_{n} \int_{0}^{\infty} d \omega \omega^{n-1} e^{-\frac{\omega}{\omega_{c}}} \frac{\sin \omega t}{\omega t} \cos \left(\frac{\omega|\mathbf{d}|}{c_{s}}\right) ;
$$

$F(t)$ commutes with $H_{\text {int }}$ and therefore could be viewed as the initial time-dependent modification of the interaction during its onset: $F(t)$ vanishes for large times as $\alpha_{n} \omega_{c}^{n} /\left(\omega_{c} t\right)^{n}$, but note that $F(0)=-H_{\text {int }}$.

The interaction Hamiltonian 10 is consistent with the results obtained [2] within a perturbative Markovian approach, for more general cases. In Figure 1, we plot the magnitude of the interaction Hamiltonian $\mathcal{H}_{\text {int }}$, defined via $H_{\text {int }}=\mathcal{H}_{\text {int }} \sigma_{x}^{1} \sigma_{x}^{2}$, as a function of the qubit-qubit separation for various $n$. At large distances the interaction decreases as $|\mathbf{d}|^{-n}$, for even $n$, and $|\mathbf{d}|^{-n-1}$, for odd $n$. This means, for instance, that for spins (as qubits) with $n=1,2$, the induced interaction decreases slower as compared to the dipole-dipole magnetic interaction; see estimates for semiconductor impurity electron spins in [2].

The decoherence terms, (5), describe quantum noise that ultimately destroys the coherent dynamics given by $H_{\text {int }}$ (and $F(t)$ ). To study the effect of these terms, we evaluate the concurrence [9] which measures the entanglement of the spin system and is monotonically related to the entanglement of formation [10]. For a mixed state of two qubits we first define the spin-flipped state, $\tilde{\rho}_{S}=\sigma_{y}^{1} \sigma_{y}^{2} \rho_{S}^{*} \sigma_{y}^{1} \sigma_{y}^{2}$,

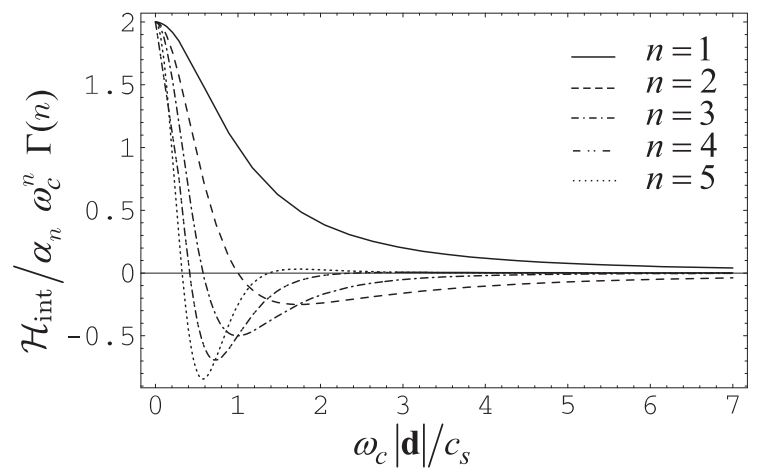

Figure 1: The magnitude of the induced interaction for the Ohmic $(n=1)$ and super-Ohmic $(n>1)$ bath models as a function of qubits' separation.

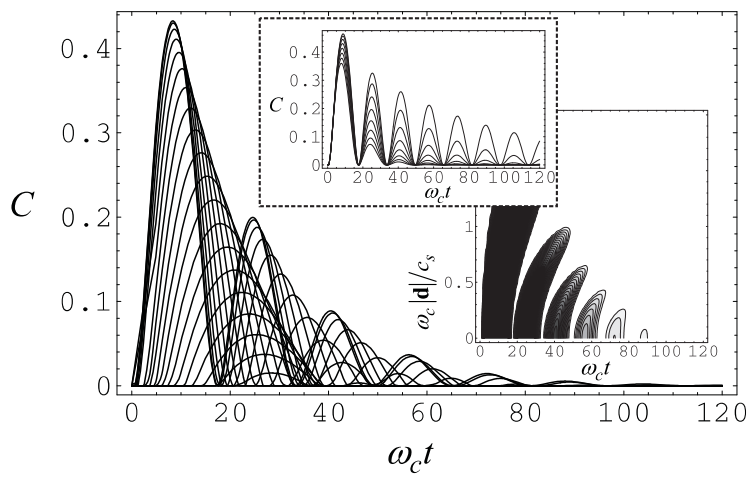

Figure 2: Concurrence as a function of time for various distances between the qubits. The right-bottom inset demonstrates the topology of the concurrence in distance-time plane. The parameters are $\alpha_{1}=1 / 20, k_{B} T / \omega_{c}=1 / 20$, $n=1$. The top inset shows dynamics for different temperatures: $80 k_{B} T / \omega_{c}=1,2,3,4,5,6,7,8$.

and then the Hermitian operator $R=\sqrt{\sqrt{\rho_{S}} \tilde{\rho}_{S} \sqrt{\rho_{S}}}$, with eigenvalues $\lambda_{i=1,2,3,4}$. The concurrence is then given [9] by

$$
C\left(\rho_{S}\right)=\max \left\{0,2 \max _{i} \lambda_{i}-\lambda_{1}-\lambda_{2}-\lambda_{3}-\lambda_{4}\right\} .
$$

In Figure 2, we plot the concurrence as a function of time and the qubit-qubit separation, for the (initially unentangled) state $|\uparrow \uparrow\rangle$, and $n=1$. The bath-mediated interaction between the qubits creates entanglement, which oscillates according to the magnitude of $H_{\text {int }}$. The same bath also damps the oscillations destroying the entanglement for larger times. The decay rate of the envelope is proportional to the temperature, as shown in the inset of Figure 2. For the corresponding dynamics of the density matrix elements see Section 5. 


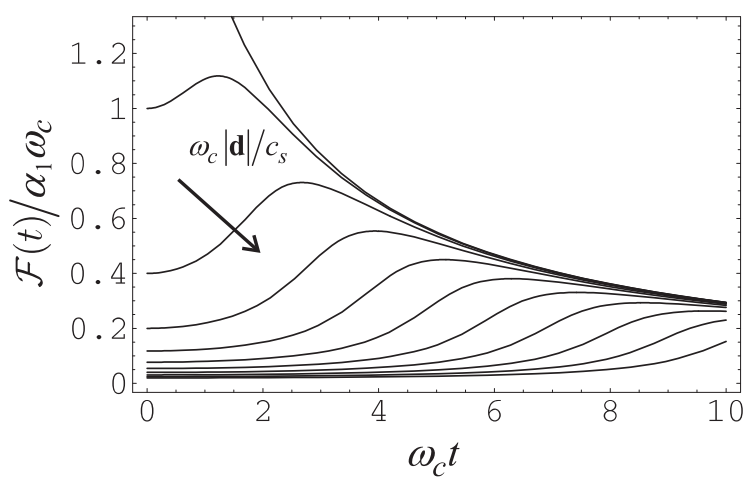

Figure 3: Initial correction to the induced interaction vs. time, for various distances between the qubits: $\omega_{c}|\mathbf{d}| / c_{s}=$ $0,1, \ldots, 10$. The Ohmic $(n=1)$ case is shown.

\section{Onset of the interaction term}

Let us now investigate in greater detail the onset of the induced interaction the time-dependence of which is given by $F(t)$. In Figure 3, we plot the magnitude defined via $F(t)=\mathcal{F}(t) \sigma_{x}^{1} \sigma_{x}^{2}$, as a function of time for various qubitqubit separations and $n=1$. The correction is initially non-monotonic, but decreases for larger times as mentioned above. The behavior for other non-Ohmic regimes is initially more complicated, however the large time behavior is similar.

It may be instructive to consider the time dependent correction, $H_{F}(t)$, to the interaction Hamiltonian during the initial evolution, corresponding to $F(t)$. Since $F(t)$ commutes with itself at different times, it generates unitary evolution according to $\exp \left[-i \int_{0}^{t} d t^{\prime} H_{F}\left(t^{\prime}\right)\right]$, with $H_{F}(t)=$ $d[t F(t)] / d t$,

$$
\begin{aligned}
H_{F}(t) & =\sigma_{x}^{1} \sigma_{x}^{2} \alpha_{n} \Gamma(n) \\
& \times\left[u\left(\omega_{c}|\mathbf{d}| / c_{s}-w_{c} t\right)+u\left(\omega_{c}|\mathbf{d}| / c_{s}+w_{c} t\right)\right]
\end{aligned}
$$

where $u(\xi)=\cos [n \arctan (\xi)] /\left[1+\xi^{2}\right]^{n / 2}$. The above expression is a superposition of two waves propagating in opposite directions. In the Ohmic case, $n=1$, the shape of the wave is simply $u(\xi)=1 /\left(1+\xi^{2}\right)$. In Figure 4 , we present the amplitude of $H_{F}(t)$, defined via $H_{F}(t)=\mathcal{H}_{F} \sigma_{x}^{1} \sigma_{x}^{2}$, as well as the sum of $H_{\text {int }}$ and $H_{F}(t)$, for $n=1$. One can observe that the "onset wave" of considerable amplitude and shape $u(\xi)$ propagates once between the qubits, "switching on" the interaction. It does not affect the qubits once the interaction has set in.

\section{Dynamics of the density matrix elements}

To understand the dynamics of the qubit system and its entanglement, let us again begin with the analysis of the

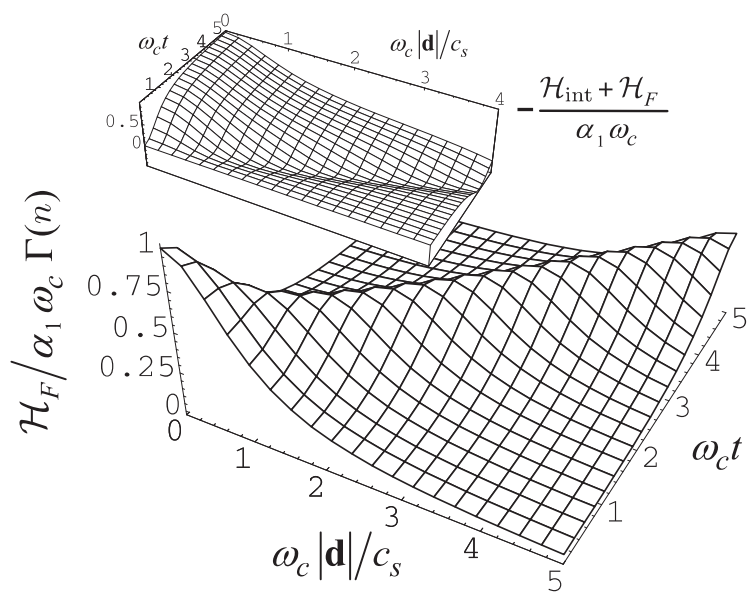

Figure 4: The magnitude of the time-dependent Hamiltonian corresponding to the initial correction as a function of time and distance. The Ohmic $(n=1)$ case is shown. The inset demonstrates the onset of the cross-qubit interaction on the same time scale.

coherent part in (4). After the interaction, $H_{\text {int }}$, has set in, it will split the system energies into two degenerate pairs, $E_{0}=E_{1}=-\mathcal{H}_{\text {int }}$ and $E_{2}=E_{3}=\mathcal{H}_{\text {int }}$. The wave function is then $|\psi(t)\rangle=\exp \left[-i H_{\text {int }} t\right]|\psi(0)\rangle$. For the initial "up-up" state, $|\psi(0)\rangle=|\uparrow \uparrow\rangle$, it develops as $|\psi(t)\rangle=|\uparrow \uparrow\rangle \cos \mathcal{H}_{\text {int }} t+|\downarrow \downarrow\rangle i \sin \mathcal{H}_{\text {int }} t$. One can easily notice that at times $t_{E}=\pi / 4 \mathcal{H}_{\text {int }}, 3 \pi / 4 \mathcal{H}_{\text {int }}, \ldots$, maximally entangled states are obtained, while at times $t_{0}=$ $0, \pi / 2 \mathcal{H}_{\text {int }}, \pi / \mathcal{H}_{\text {int }}, \ldots$, the entanglement vanishes; these special times can also be seen in Figure 2.

The bath also induces decoherence that enters via (5). The result for the entanglement is that the decaying envelope function is superimposed onto the coherent dynamics described above. The magnitudes of the first and subsequent peaks of the concurrence are determined only by this function. As temperature increases, the envelope decays faster resulting in lower values of the concurrence, see the inset in Figure 2.

Note also that generation of entanglement is possible only provided that the initial state is a superposition of the eigenvectors of the induced interaction with more than one eigenvalue (for pure initial states). For example, the initial state $(|\downarrow \uparrow\rangle+|\uparrow \downarrow\rangle) / \sqrt{2}$ in our case would only lead to the destruction of entanglement, i.e., monotonically decreasing concurrence, similar to the results in [11].

Since $H_{S}=0$, there is no relaxation by energy transfer in the system, and the exponentials in (4), with (5), suppress only the off-diagonal matrix elements, i.e., those with $\lambda \neq \lambda^{\prime}$. It happens, however that at large times the $\mathbf{d}$ dependence is not important in (5) and $\operatorname{Re} \mathfrak{L}_{\lambda \lambda^{\prime}}(t \rightarrow \infty)$ vanishes for certain values of $\lambda \neq \lambda^{\prime}$. In the basis of the 


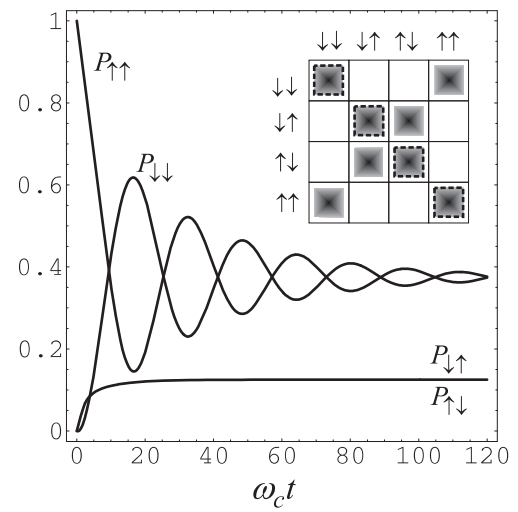

Figure 5: Dynamics of the occupation probabilities for the states $|\uparrow \uparrow\rangle,|\uparrow \downarrow\rangle,|\downarrow \uparrow\rangle$, and $|\downarrow \downarrow\rangle$. The parameters are the same as in Figure 1. The inset shows the structure of the reduced density matrix (the non-shaded entries are zeros).

qubit-bath interaction, $\sigma_{x}^{1} \sigma_{x}^{2}$, the limiting $t \rightarrow \infty$ density matrix for our initial state $(|\uparrow \uparrow\rangle)$ is $\frac{1}{4}+\frac{1}{4}|+-\rangle\langle-+|+$ $\frac{1}{4}|-+\rangle\langle+-|$, which takes the form

$$
\rho(t \rightarrow \infty) \rightarrow \frac{1}{8}\left(\begin{array}{cccc}
3 & 0 & 0 & -1 \\
0 & 1 & 1 & 0 \\
0 & 1 & 1 & 0 \\
-1 & 0 & 0 & 3
\end{array}\right)
$$

in the basis of states $|\uparrow \uparrow\rangle,|\uparrow \downarrow\rangle,|\downarrow \uparrow\rangle$, and $|\downarrow \downarrow\rangle$. The significance of such results, see also [3], is that in the present model not all the off-diagonal matrix elements are suppressed by decoherence, even though the concurrence of this mixed state is zero.

The probabilities for the qubits to occupy the states $|\uparrow \uparrow\rangle$, $|\uparrow \downarrow\rangle,|\downarrow \uparrow\rangle$, and $|\downarrow \downarrow\rangle$ are presented in Figure 5. For the initial state $|\uparrow \uparrow\rangle$, only the diagonal and inverse-diagonal matrix elements are affected, and the system oscillates between the two states $|\uparrow \uparrow\rangle$ and $|\downarrow \downarrow\rangle$, as mentioned earlier in the description of the coherent dynamics, while decoherence dampens these oscillations down. In addition, decoherence actually raises the other two diagonal elements to a certain level, see (14). The dynamics of the inverse-diagonal density matrix elements is shown in Figure 6.

\section{Conclusion}

To summarize, we studied the initial stages of the crossqubit interaction induced by a thermal bosonic bath. It was shown that thermal environment can create a sufficiently large entanglement for quantum control, though it is erased for larger times. The dynamics of the entanglement and the density matrix elements have been investigated.

This research was supported by the NSF under grant DMR0121146.

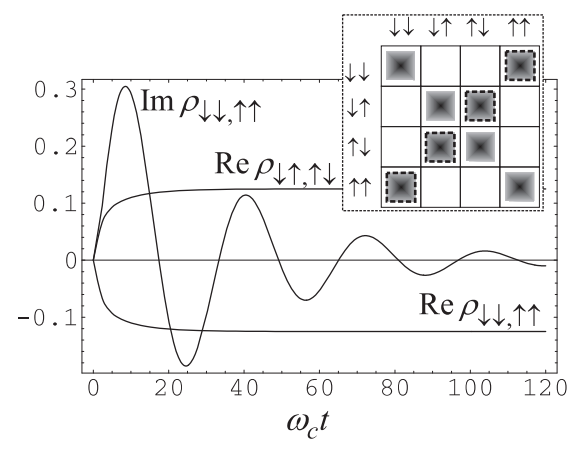

Figure 6: Dynamics of the off-diagonal matrix elements for the same system as in Figure 5.

\section{References}

[1] D. Solenov, D. Tolkunov, and V. Privman, Phys. Lett. A (in print), cond-mat/0511680

[2] D. Solenov, D. Tolkunov, and V. Privman, cond-mat/0605278

[3] D. Braun, Phys. Rev. Lett. 89, 277901 (2002).

[4] D. Porras and J. I. Cirac, Phys. Rev. Lett. 92, 207901 (2004).

[5] M. A. Ruderman and C. Kittel, Phys. Rev. 96, 99 (1954); T. Kasuya, Prog. Theor. Phys. 16, 45 (1956); K. Yosida, Phys. Rev. 106, 893 (1957); V. Privman, I. D. Vagner, and G. Kventsel, Phys. Lett. A 239, 141 (1998); C. Piermarocchi, P. Chen, L. J. Sham, and D. G. Steel, Phys. Rev. Lett. 89, 167402 (2002); D. Mozyrsky, A. Dementsov, and V. Privman, Phys. Rev. B 72, 233103 (2005); Y. Rikitake and H. Imamura, Phys. Rev. B 72, 033308 (2005).

[6] N. J. Craig, J. M. Taylor, E. A. Lester, C. M. Marcus, M. P. Hanson, and A. C. Gossard, Science 304, 565 (2004); J. M. Elzerman, R. Hanson, L. H. Willems van Beveren, B. Witkamp, L. M. K. Vandersypen, and L. P. Kouwenhoven, Nature 430, 431 (2004); M. R. Sakra, H. W. Jiang, E. Yablonovitch, and E. T. Croke, Appl. Phys. Lett. 87, 223104 (2005).

[7] A. J. Leggett, S. Chakravarty, A. T. Dorsey, M. P. A. Fisher, A. Garg, and W. Zwerger, Rev. Mod. Phys. 59, 1 (1987).

[8] V. Privman, Modern Phys. Lett. B 16, 459 (2002).

[9] S. Hill and W. K. Wootters, Phys. Rev. Lett. 78, 5022 (1997); W. K. Wootters, Phys. Rev. Lett. 80, 2245 (1998).

[10] C. H. Bennett, D. P. DiVincenzo, J. Smolin, and W. K. Wootters, Phys. Rev. A 54, 3824 (1996).

[11] T. Yu and J. H. Eberly, Phys. Rev. B 68, 165322 (2003); T. Yu and J. H. Eberly, Phys. Rev. Lett. 93, 140404 (2004). 